\title{
Cytokine response during non-cerebral and cerebral malaria: evidence of a failure to control inflammation as a cause of death in African adults
}

Yakhya Dieye, Babacar Mbengue, Shobha Dagamajalu, Mouhamadou M Fall, Mun Fai Loke, Cheikh M Nguer, Alassane Thiam, Jamuna Vadivelu, Alioune Dieye

Background. With 214 million cases and 438,000 deaths in 2015, malaria remains one of the deadliest infectious diseases in tropical countries. Several species of the protozoan Plasmodium cause malaria. However, almost all the fatalities are due to Plasmodium falciparum, a species responsible for the severest cases including cerebral malaria. Immune response to Plasmodium falciparum infection is mediated by the production of pro-inflammatory cytokines, chemokines and growth factors whose actions are crucial for the control of the parasites. Following this response, the induction of anti-inflammatory immune mediators downregulates the inflammation thus preventing its adverse effects such as damages to various organs and death. Methods. We performed a retrospective, nonprobability sampling study using clinical data and sera samples from patients, mainly adults, suffering of non-cerebral or cerebral malaria in Dakar, Sénégal. Healthy individuals residing in the same area were included as controls. We measured the serum levels of 29 biomarkers including growth factors, chemokines, inflammatory and anti-inflammatory cytokines. Results. We found an induction of both pro- and anti-inflammatory immune mediators during malaria. The levels of pro-inflammatory biomarkers were higher in the cerebral malaria than in the non-cerebral malaria patients. In contrast, the concentrations of anti-inflammatory cytokines were comparable in these two groups or lower in CM patients. Additionally, four pro-inflammatory biomarkers were significantly increased in the deceased of cerebral malaria compared to the survivors. Regarding organ damage, kidney failure was significantly associated with death in adults suffering of cerebral malaria.

Conclusions. Our results suggest that a poorly controlled inflammatory response determines a bad outcome in African adults suffering of cerebral malaria. 
1 Cytokine Response during Non-cerebral and Cerebral Malaria: evidence of a failure to 2 control inflammation as a cause of death in African adults

3 Yakhya Dieye ${ }^{1 *}$, Babacar Mbengue ${ }^{2,3}$, Shobha Dagamajalu ${ }^{4}$, Mouhamadou Mansour Fall ${ }^{5}$, Mun

4 Fai Loke ${ }^{4}$, Cheikh Momar Nguer ${ }^{6}$, Alassane Thiam ${ }^{3}$, Jamuna Vadivelu ${ }^{4}$, and Alioune Dieye ${ }^{2,3}$

5

$6{ }^{1}$ Vice-chancellor's Office, University of Malaya, Kuala Lumpur, Malaysia

72 Département d'Immunologie, Faculté de Médicine, de Pharmacie et d'Odontostomatologie,

8 Université Cheikh Anta Diop, Dakar, Sénégal

$9 \quad{ }^{3}$ Unité d'Immunogénétique, Institut Pasteur de Dakar, Sénégal

$10{ }^{4}$ Department of Medical Microbiology, Faculty of Medicine, University of Malaya, Kuala

11 Lumpur, Malaysia

125 Service de Réanimation, Hôpital Principal de Dakar, Sénégal

136 Département Génie Chimique et Biologie Appliquée, École Supérieure Polytechnique,

14 Université Cheikh Anta Diop, Dakar, Sénégal

$15 *$ Corresponding author:

16 Yakhya Dieye

17 Permanent address: Département Génie Chimique et Biologie Appliquée, École Supérieure

18 Polytechnique, Université Cheikh Anta Diop, BP 5085 Dakar, Sénégal

19 E-mail: y.dieye@laposte.net 
21 Abstract

22 Background. With 214 million cases and 438,000 deaths in 2015, malaria remains one of the deadliest infectious diseases in tropical countries. Several species of the protozoan Plasmodium cause malaria. However, almost all the fatalities are due to Plasmodium falciparum, a species responsible for the severest cases including cerebral malaria. Immune response to Plasmodium falciparum infection is mediated by the production of pro-inflammatory cytokines, chemokines and growth factors whose actions are crucial for the control of the parasites. Following this response, the induction of anti-inflammatory immune mediators downregulates the inflammation thus preventing its adverse effects such as damages to various organs and death.

Methods. We performed a retrospective, nonprobability sampling study using clinical data and sera samples from patients, mainly adults, suffering of non-cerebral or cerebral malaria in Dakar, Sénégal. Healthy individuals residing in the same area were included as controls. We measured the serum levels of 29 biomarkers including growth factors, chemokines, inflammatory and antiinflammatory cytokines.

Results. We found an induction of both pro- and anti-inflammatory immune mediators during malaria. The levels of pro-inflammatory biomarkers were higher in the cerebral malaria than in the non-cerebral malaria patients. In contrast, the concentrations of anti-inflammatory cytokines were comparable in these two groups or lower in CM patients. Additionally, four proinflammatory biomarkers were significantly increased in the deceased of cerebral malaria compared to the survivors. Regarding organ damage, kidney failure was significantly associated with death in adults suffering of cerebral malaria.

42 Conclusions. Our results suggest that a poorly controlled inflammatory response determines a bad outcome in African adults suffering of cerebral malaria. 


\section{Introduction}

45

46

47

Despite a decade of sustained efforts that have substantially reduced mortality and morbidity due to malaria, this disease continues to represent an important health concern in tropical countries (White et al. 2014). According to the World Health Organization (WHO), there were 214 million cases of malaria worldwide in 2015, which resulted in 438,000 deaths (WHO 2015). Ninety percent of the victims were from Africa, $74 \%$ being children under 5 years of age. Malaria is endemic in many sub-Saharan African countries. However, there are disparities between (WHO 2015) and even within countries (Espie et al. 2015) regarding the transmission of the disease. In many rural areas where the local environment favors the development of the mosquito vector and its interactions with humans, transmission of malaria is high and perennial (Trape et al. 2014). In contrast, in other areas including urban zones, the transmission of malaria is low to moderate and seasonal (White et al. 2014). Individuals living in regions of high and stable transmission progressively acquire immunity after experiencing and surviving to several infections (Olliaro 2008). This immunity protects against severe, life-threatening cases of malaria but does not confer a sterile protection (Doolan et al. 2009). In these areas, clinical malaria occurs in young children while healthy carriage of the parasite is common in adults. Adults who die of malaria typically are pregnant women or non-immune individuals from low transmission zones.

There was a decline of $18 \%$ and $48 \%$ of global malaria cases and deaths respectively between 2000 and 2015. This success was primarily due to a drastic reduction of malaria transmission by widespread use of insecticide-treated bednets and the availability of artemisinin-based treatments (Bhatt et al. 2015; White et al. 2014). The decline of malaria is expected to continue with the support of the WHO Global Technical Strategy for malaria 2016-2030 that aims to reduce its 
67 global incidence and mortality by at least $90 \%$ by 2030 (WHO 2015). However, a continuous

68 decrease of malaria prevalence and especially a sustained reduction of transmission in currently

69 holoendemic zones may result, in the future, in an increase of the number of adults susceptible to

70 severe cases. Such an unwanted but possible scenario potentially represents a future public health

71 concern in sub-Saharan African countries. Several species of the protozoan Plasmodium cause

72 malaria. However, almost all the deaths are due to $P$. falciparum, a species that causes the

73 severest cases including cerebral malaria (Storm \& Craig 2014). In response to P. falciparum

74 infection, a robust immune inflammatory response takes place. An important component of this

75 response is the production of inflammatory immune mediators whose actions are crucial for the control of the parasites (Deloron et al. 1994; Lyke et al. 2004; Sarthou et al. 1997). This

77 inflammatory response is rapidly followed by the production of anti-inflammatory cytokines that downregulate the inflammation preventing detrimental immune reactions (Kurtzhals et al. 1998;

Peyron et al. 1994; Walther et al. 2005). Therefore, the immune response to P. falciparum infection includes a subtle balance of pro- and anti-inflammatory immune mediators (Crompton et al. 2014; Frosch \& John 2012). A rupture of this balance is at the basis of the events that lead to organ damage and death (Crompton et al. 2014). The pathogenesis of severe malaria and its associated mortality have been widely studied in children. In contrast, there are less investigations that addressed these aspects of the disease in adults, in particular from Africa (Olliaro 2008). In this study, we performed a retrospective analysis of the available clinical data and of the immune response of malaria patients, mainly adults, admitted at the Hôpital Principal de Dakar, Sénégal. Malaria is endemic in several areas in Sénégal. However, the capital city Dakar and its surroundings constitute a zone of low prevalence of malaria with a seasonal transmission. We report the analysis of the serum levels of cytokines, chemokines and growth 
90 factors in control individuals and in patients suffering of non-cerebral (NCM) or cerebral malaria

91 (CM). All the CM patients were adults and included deceased and survivors enabling to gain

92 insights into the effect of the analysed biomarkers in the outcome of the disease.

93 Materials and methods

94 Study population, ethics, consent and permissions

95 This study was performed on serum samples from patients diagnosed with malaria at the Hôpital

96 Principal de Dakar, Sénégal between October 2012 and December 2014 (Torrentino-Madamet et

97 al. 2014). The samples were taken after written consents from the patients or their accompanying

98 family members. The controls corresponded to samples obtained from healthy volunteers residing in Dakar. This study was approved by the Université Cheikh Anta Diop de Dakar's

100 institutional research ethics committee (Protocol N 001/2015/CER/UCAD). Venous blood

101 samples were collected in Vacutainer ${ }^{\circledR}$ ACD tubes (Becton Dickinson, Rutherford, NJ, USA)

102 prior to patient treatment. Plasmodium presence and density in blood samples were determined

103 by microscopic examination of thin blood smears stained with a 10\% May-Grünwald Giemsa

104 solution (SigmaR, St-Louis, MO, USA). P. falciparum was the only species found. Blood 105 parameters were determined at the hospital's clinical laboratory. The following criteria were 106 used for enrollment into the two groups of malaria patients. Life-threatening CM was defined 107 following the WHO criteria as the presence of $P$. falciparum in blood smears accompanied by a 108 coma with no other cause of cerebral symptoms. NCM cases were defined by fever and presence 109 of P. falciparum in blood smear, without other infections or symptoms of severe malaria as 110 defined by the WHO (WHO 2000). CM patients were treated according to a protocol based on

111 the Senegalese national recommendations that consisted of intramuscular administrations of 20

$112 \mathrm{mg} / \mathrm{kg}$ quinine every eight hours. NCM patients were treated with oral administration of 20 
$113 \mathrm{mg} / \mathrm{kg}$ of artesunate derivates or quinine. Secondary samples analyzed in this study corresponded

114 to blood taken from survivors of CM (14 individuals) before patient release from the hospital (1-

11515 days after admission).

\section{Biomarker measurement}

117 Serum biomarkers were measured using a Milliplex MAP kit for human cytokine/chemokine

118 magnetic bead panel (catalogue \# HCYTMAG-60K-PX29, EMD Millipore Corporation,

119 Billerica, MA, USA) according to the recommendations of the manufacturer. The levels of 29

120 biomarkers were measured in each sample including interleukin (IL)-1 $\alpha$, IL-1 $\beta$, IL-1RA, IL-2,

IL-3, IL-4, IL-5, IL-6, IL-7, IL-8, IL-10, IL-12 (p40), IL-12 (p70), IL-13, IL-15, IL-17,

122 interferon (IFN) $\alpha 2$, IFN $\gamma$, IFN-inducible protein 10 (IP-10, CXCL-10), epidermal growth factor

123 (EGF), eotaxin, granulocyte colony-stimulating factor (G-CSF), granulocyte-macrophage

124 colony-stimulating factor (GM-CSF), tumor necrosis factor (TNF) $\alpha$, TNF $\beta$, monocyte

125 chemotactic protein (MCP)-1, macrophage inflammatory protein (MIP)-1 $\alpha$, MIP-1 $\beta$, and

126 vascular endothelial growth factor (VEGF). The measurements were performed in $25 \mathrm{ul}$ of

127 undiluted serum samples on one 96 well plate. Each well contained fluorescent-coded magnetic

128 microbeads coated with analyte-specific capture antibodies to simultaneously measure the

129 biomarkers in a specimen. Seven standards and two quality-control (QC) samples were included

130 and measured in duplicate. The QC samples corresponded to mixtures with two values (high and

131 low) for each biomarker. After the capture of the biomarkers, the beads were washed, incubated

132 with biotinylated antibodies and then with streptavidin-PE. Excitation and fluorescence

133 acquisition from the beads were performed using a Luminex $200^{\mathrm{TM}}$ equipped with an

134 XPONENT ${ }^{\mathrm{TM}}$ software version 3.1 (Luminex, Austin, TX, USA) that calculated the

135 concentrations of the biomarkers by extrapolating the mean fluorescence intensity (MFI) to a 5- 
136 parameter weighted logistic regression curve from the standards. Any measurement below the

137 detection limit was given a value of 0 for the corresponding analyte. For most of the biomarkers,

138 the majority of the samples had detectable values. For IL-2, IL-3, IL-4, IL12p40, IL-13 and

139 TNF $\beta$, the small number of samples with detectable MFI did not permit meaningful statistical

140 analyses. These biomarkers were excluded from the statistical analyses. The MFI for one G-CSF

141 and two IL-1RA samples were above the value of the highest standard whose concentration was

$14210,000 \mathrm{pg} / \mathrm{ml}$. These samples were arbitrarily assigned concentrations above the highest

143 standard. The G-CSF sample was assigned a concentration of $11,000 \mathrm{pg} / \mathrm{ml}$. The two IL-1RA

144 samples were attributed concentrations of $11,000 \mathrm{pg} / \mathrm{ml}$ and $12,000 \mathrm{pg} / \mathrm{ml} \mathrm{respectively} \mathrm{according}$

145 to the values of their MFI. Treating the samples above the highest standard in this way, rather

146 than excluding them, allowed to take them into account when determining the median values for

147 G-CSF and IL-1RA.

\section{Statistical analyses}

149 The statistical analyses were carried out using the IBM SPSS 22.0 software. Non-parametric 150 tests were used to compare the levels of biomarker and their correlation with other variables

151 across different groups. Mann-Whitney U test and Kruskal-Wallis one-way ANOVA were used

152 to compare data across two and three groups respectively. Wilcoxon matched pairs signed rank

153 test was used to compare biomarker levels in sera from CM patients at admission and at their

154 release from the hospital. Correlation tests were performed using Spearman's Rho rank test.

155 Pearson Chi-Square was used to test association of organ failure with outcome in CM patients.

156 Benjamini-Hochberg correction was used for multiple testing adjustment. For all the statistical

157 analyses, a $p$ value $<0.05$ was considered as significant except when multiple testing adjustment

158 was used, in which cases significant $p$ values depended on the critical values from the 
159 Benjamini-Hochberg correction. The biomarker profiles were determined as previously

160 described (da Costa et al. 2014). In brief, the median value in the global population (CT + NCM

$161+\mathrm{CM}$ ) was calculated for each biomarker and used as a cut-off to determine the percentage of

162 individuals that had "high" (above the median value) and "low" (below the median value) levels

163 of biomarker in the CT, NCM and CM groups. An ascendant biomarker profile was then

164 constructed in the CT group by assembling the biomarkers from the one having the smallest

165 percentage of high producers to the one having the largest. The resulting ascendant curve was

166 used as a reference to visualize the variation of the percentage of high producers of biomarkers in

167 the other groups (Fig. 1). In addition to showing the differences in the percentage of high

168 producers (descriptive statistics), the biomarker profiles also indicate the analytes (hatched bars)

169 for which there was a significant difference in adjusted Mann-Whitney U pairwise comparison

170 (inferential statistics).

\section{Results}

\section{Study population and clinical data}

173 We performed a retrospective, nonprobability sampling study using sera samples from healthy

174 individuals and from patients admitted at the Hôpital Principal de Dakar, Sénégal. The cohort

175 included 17 and 27 subjects diagnosed with NCM and CM respectively, and 18 healthy controls

176 (CT) (Table 1). The three groups of individuals were comparable in age and gender, and were

177 mainly composed of adults (Table 1). Subjects below 15 years of age included six children aged

178 5-13 diagnosed with NCM. Several clinical and blood parameters existed but were not recorded

179 for all the individuals preventing reliable statistical analyses. Available data showed, as

180 expected, hemoglobin levels comparable in the CT and NCM individuals while significantly

181 lower in the CM patients (Table 1). Additionally, parasitemia was comparable between the NCM 
182 and CM groups (Table 1). Regarding organ defect in the CM group, kidney failure was the most

183 frequent (13/27) followed by liver, hematologic and respiratory malfunction, while

184 hemodynamic failure was rare (Table 1). All the NCM patients were successfully treated, while 185 9/27 CM subjects died.

\section{Levels of inflammatory but not of anti-inflammatory biomarkers were higher in CM than}

187

188

\section{in NCM patients}

To analyze the production of immune mediators during malaria, we measured the serum levels of 29 biomarkers including growth factors, chemokines, inflammatory and anti-inflammatory cytokines. We determined the ascendant biomarker profile of the CT group as previously described (da Costa et al. 2014) and plotted the resulting curve on the profiles of the NCM and CM patients (Fig. 1). The biomarker profiles display comparison of the proportion of individuals with levels of analytes above the global median between the controls and the malaria patients (Figs. 1B and 1C). Additionally, analytes that significantly differ in Mann-Whitney U pairwise comparison after Benjamini-Hochberg multiple testing adjustment are shown in the biomarker profiles (Figs. 1B and 1C, hatched bars). Several analytes were significantly higher in malaria patients (NCM and/or CM) than in CT individuals (Figs. 1 and 2). These biomarkers included most of the pro-inflammatory cytokines and chemokines tested (IL-1 $\alpha$, IL-6, IL-8, IL-12p70, IL15, IL-17A, IP-10, TNF $\alpha$, IFN $\alpha 2$, IFN $\gamma$, MIP-1 $\alpha$, MIP-1 $\beta$ and MCP-1) and the antiinflammatory cytokines IL-10 and IL-1RA. The induction of both inflammatory and antiinflammatory immune mediators that aims to respond to the infection while controlling the level of inflammation in order to prevent damages to host organs has been well documented in malaria patients (Frosch \& John 2012). Also, we observed an induction of Th1 (IL-12, IFN $\gamma$ and TNF $\alpha$ ) and Th2 (IL-10) biomarkers in both NCM and CM patients. Since only the control group 
205 included children, we performed pairwise comparisons after removing subjects below 15 years 206 of age. We did not find differences in the analytes that significantly differed whether children

207 were included or not except for IFN 22 that lost significance in the CT/NCM comparison (not

208 shown). Besides individual biomarkers, several analytes were significantly positively correlated

209 consistent with the immune response during malaria that mobilizes several cytokines,

210 chemokines and growth factors (Supplementary File 1). Next, we analyzed the difference of

211 cytokine levels between the NCM and CM patients. Most of the biomarkers induced during

212 malaria were higher in $\mathrm{CM}$ than in the NCM individuals (Fig. 3). However, after Benjamini-

213 Hochberg adjustment for multiple tests (Table 2), only the levels of the pro-inflammatory IL-6

214 and IL-8, and of IL-1RA, an antagonist of IL-1 reached statistical significance (Fig. 3 and Table

215 2). These results indicate an inflammatory response of higher magnitude in CM compared to

216 NCM patients as previously mentioned in several reports (Crompton et al. 2014). In contrast, the

217 level of IL-10 was not significantly different between NCM and CM patients (Fig. 3).

218 Additionally, the level of IL-5, a Th2 anti-inflammatory cytokine, was significantly lower in CM

219 than in NCM patients (Fig. 3 and Table 2) as was the ratios of IL-5 to the pro-inflammatory

220 biomarkers TNF $\alpha$, IP-10 and IL-8 (not shown). These results suggest that the level of anti-

221 inflammatory response did not match the strength of the inflammatory cytokine response in the

222 CM patients.

223

224

225

226

227

\section{Levels of inflammatory biomarkers were lower in survivors than in deceased of CM}

Failure to control inflammation is proposed as one of the mechanisms leading to CM, which is consistent with the difference we observed between NCM and CM patients. To further analyze the effect of the inflammatory biomarkers, we compared the levels of analyte between the survivors $(n=18)$ and the deceased $(n=9)$ of CM. Interestingly, after multiple testing 
228 adjustment (Table 3), there were four biomarkers whose levels significantly differed between the

229 two groups. All were pro-inflammatory analytes (Eotaxin, IL-15, MCP-1 and TNF $\alpha$ ) that were

230 significantly lower in the survivors than in the deceased of CM (Fig. 4). These results suggest

231 that the cause of death involved an inflammatory response of high magnitude that was not

232 properly controlled. To analyze possible effects of the inflammatory response in tissue damage,

233 we compared the failure of different organs between survivors and deceased CM patients.

234 Kidney failure was significantly associated with patient's death $\left(\chi^{2}[1, \mathrm{~N}=27]=8.98, p=0.003\right.$;

235 Effect Size $=0.58$ ) while the occurrence of neurological, respiratory, liver, hematologic and

236 hemodynamic failures were comparable between the two groups. We further attempted to

237 correlate the biomarker levels with organ failure. Kidney failure showed significant moderate to

238 strong positive correlations with several chemokines and pro-inflammatory cytokines (Table 4),

239 while respiratory, hematological and liver failure displayed weak positive correlations with 5, 5

240 and 1 biomarkers respectively (not shown).

241 Variation of biomarker levels before and after cerebral malaria treatment

242 Analysis of biomarker profiles in malaria patients before and after treatment provides valuable

243 information on immune mediators that are induced during malaria. We compared the levels of

244 biomarker between the time of emergency admission and of hospital release in 14 CM patients

245 (Table 5). Wilcoxon rank test showed 7 biomarkers (G-CSF, IL-10, IL-1 $\alpha$, IL-8, IP-10, MCP-1,

$246 \mathrm{TNF} \alpha$ ) that were significantly different between the two time points after Benjamini-Hochberg

247 adjustment. All these biomarkers were lower in the second samples confirming the induction of

248 different types of immune mediators including growth factor (G-CSF), inflammatory (TNF $\alpha$, IL-

$2491 \alpha$, IP-10), anti-inflammatory (IL-10) and chemokines (IL-8, MCP-1) during immune response

250 to malaria (Table 5). 


\section{Discussion}

252 Inflammation and outcome of cerebral malaria

253 In this study, we performed a retrospective analysis of 18 controls, and of 17 and $27 \mathrm{NCM}$ and

254 CM patients respectively. The CM patients included 18 survivors and 9 (30\%) deceased subjects,

255 a proportion similar to the highest mortality rates reported for CM. Beside neurological defect,

256 kidney failure was the most frequent organ malfunction in CM patients and was correlated with

257 death. Analysis of the cytokine response showed a strong induction of pro- and anti-

258 inflammatory biomarkers in malaria patients. However, the magnitude of this response was

259 significantly higher in CM than in NCM patients for inflammatory biomarkers while it was

260 comparable in the two groups for the anti-inflammatory cytokines. Additionally, comparison of

261 the biomarkers in the survivors versus the deceased of CM showed four pro-inflammatory

262 analytes that were significantly higher in the deceased patients. Altogether, our results suggest a

263 scenario in which a strong inflammatory response that was not properly contained led to organ

264 failure and death during CM.

265 The involvement of the inflammatory response in the pathogenesis of severe malaria,

266 including CM, is well documented (Clark et al. 2008). The balance of pro- and anti-

267 inflammatory cytokines, chemokines and growth factors is key to controlling parasite

268 development without damages to host organs. Regarding the individual biomarkers, we found

269 pro-inflammatory immune mediators increased in malaria patients with levels of IL-6 and IL-8

270 higher in CM compared to NCM individuals, and of eotaxin, IL-15, MCP-1 and TNF $\alpha$ elevated

271 in deceased compared to survivors of CM. The association of these cytokines and chemokines

272 with malaria severity and/or poor outcome have been described before (Clark et al. 2008). TNF $\alpha$

273 is one of the first recognized pro-inflammatory biomarkers that play important role during 
274 malaria. With other Th1 type cytokines IL-1, IL-12 and IFN $\gamma$ it contributes to the control of the 275 infection (Schofield \& Grau 2005). However, elevated levels of TNF $\alpha$ were associated with 276 disease severity in both children and adults (Prakash et al. 2006; Thuma et al. 2011) and can 277 discriminate between SM and UM (Mahanta et al. 2015). Similarly, IL-8 (Berg et al. 2014; Lyke 278 et al. 2004), IL-6 (Jakobsen et al. 1994; Lyke et al. 2004), IL-15 (Hu 2013; Ong'echa et al. 2011)

279 280

and MCP-1 (MacMullin et al. 2012; Quelhas et al. 2012) were reported as increased during malaria.

In contrast to TNF $\alpha$, IL-6, IL-8, IL-15 and MCP-1, eotaxin was not often mentioned in previous malaria studies. Interestingly, the level of eotaxin was higher in the CT individuals than in the malaria patients but the difference lost statistical significance after multiple testing adjustment. A significantly lower level of eotaxin was reported by Requena et $a l$. in pregnant women exposed to malaria when compared to controls residing in malaria-free areas (Requena et al. 2014). Additionally, eotaxin was found as a negative predictor of hemoglobin level in children with SMA (Ong'echa et al. 2011). These observations suggest that eotaxin is dowregulated during malaria and that it could be involved in pathogenesis. Eotaxin is a Th2-type chemokine that mediates eosinophil development and recruitment in host tissues (Pope et al. 2001; Queto et al. 2010). Eotaxin is an important biomarker of allergic diseases (Pope et al. 2001) and polymorphism of its encoding gene influence total serum IgE level (Batra et al. 2007; Wang et al. 2007). The role played by $\operatorname{IgE}$ in response to malaria infection is controversial with some studies claiming a protective function (Bereczky et al. 2004; Farouk et al. 2005) while other associating IgE with disease severity (Perlmann et al. 1994; Perlmann et al. 1997; SekaSeka et al. 2004). However, a recent study in a mouse model of experimental CM showed that animals genetically deficient for IgE or for the high affinity receptor for IgE were less 
297 susceptible to CM (Porcherie et al. 2011) supporting a role of IgE in the development of CM.

298 The same study showed that CM pathogenesis was mediated by neutrophils expressing the high

299 affinity receptor for IgE that homed to the brain and locally induced high levels of pro-

300 inflammatory cytokines (Porcherie et al. 2011). Whether this function could be translated to

301 human is unknown. However, a recent study reported an elevated neutrophil count that

302 correlated with expression levels of the pro-inflammatory mediators IL-1 $\beta$ and IL-8 in human

303 severe malaria (Mahanta et al. 2015). Altogether, these observations support the hypothesis that

304 elevated levels of eotaxin result in higher production of $\operatorname{IgE}$ and deleterious effects during human

305 malaria. If this is the case, the downregulation of eotaxin observed previously (Requena et al.

306 2014) and in this study might be a mechanism that protects against the damages caused by $\operatorname{IgE}$

307 during malaria. This hypothesis is consistent with higher levels of eotaxin observed in deceased

308 compared to survivors of CM. However it needs to be tested in other studies.

309

310

311

312

313

314

315

316

317

318

319

Besides eotaxin, IL-5, another Th2 type cytokine displayed a remarkable profile in this study with its level significantly decreased in CM compared to NCM patients (Fig. 3). IL-5 is a regulatory cytokine that cooperates with eotaxin in the development and recruitment of eosinophils (Nussbaum et al. 2013). A previous study reported elevated levels of IL-5 in mild compared to severe malaria patients (Prakash et al. 2006) suggesting a protective role of this cytokine. This hypothesis is consistent with a recent report of a mouse study demonstrating a protection of rodent against experimental CM by IL-33 treatment (Besnard et al. 2015). The protection against CM was mediated by IL-5 independently of eosinophils, implying a mechanism that does not involve eotaxin.

In conclusion, our study confirms previously reported inflammatory response during malaria. Our findings support the idea of a strong induction of pro-inflammatory immune 
320

321

322

323

324

325

326

327

328

329

330

331

332

333

334

335

336

337

338

339

340

341

342

343

344

345

346

347

348

349

350

351

352

353

354

355

356

mediators that was not matched by the production of regulatory, anti-inflammatory biomarkers

as the cause of death during CM. Additionally, our results suggests the involvement of eotaxin

and of IL-5 in CM development and outcome.

\section{Acknowledgements}

We would like to thank Theresa Wan-Chen Yap for her help in the dosage of the biomarkers. We are grateful to Dr Becaye Fall, Dr Pape Samba Fall and Dr Ronald Perraut for their constructive

suggestions and for stimulating discussions.

\section{References}

Batra J, Rajpoot R, Ahluwalia J, Devarapu SK, Sharma SK, Dinda AK, and Ghosh B. 2007. A hexanucleotide repeat upstream of eotaxin gene promoter is associated with asthma, serum total IgE and plasma eotaxin levels. J Med Genet 44:397-403. 10.1136/jmg.2006.046607

Bereczky S, Montgomery SM, Troye-Blomberg M, Rooth I, Shaw MA, and Farnert A. 2004. Elevated antimalarial IgE in asymptomatic individuals is associated with reduced risk for subsequent clinical malaria. Int J Parasitol 34:935-942. 10.1016/j.ijpara.2004.04.007

Berg A, Patel S, Gonca M, David C, Otterdal K, Ueland T, Dalen I, Kvaloy JT, Mollnes TE, Aukrust P, and Langeland N. 2014. Cytokine network in adults with falciparum Malaria and HIV-1: increased IL-8 and IP-10 levels are associated with disease severity. PLoS One 9:e114480. 10.1371/journal.pone.0114480

Besnard AG, Guabiraba R, Niedbala W, Palomo J, Reverchon F, Shaw TN, Couper KN, Ryffel B, and Liew FY. 2015. IL-33-mediated protection against experimental cerebral malaria is linked to induction of type 2 innate lymphoid cells, M2 macrophages and regulatory T cells. PLoS Pathog 11:e1004607. 10.1371/journal.ppat.1004607

Bhatt S, Weiss DJ, Cameron E, Bisanzio D, Mappin B, Dalrymple U, Battle KE, Moyes CL, Henry A, Eckhoff PA, Wenger EA, Briet O, Penny MA, Smith TA, Bennett A, Yukich J, Eisele TP, Griffin JT, Fergus CA, Lynch M, Lindgren F, Cohen JM, Murray CL, Smith DL, Hay SI, Cibulskis RE, and Gething PW. 2015. The effect of malaria control on Plasmodium falciparum in Africa between 2000 and 2015. Nature 526:207-211. 10.1038/nature15535

Clark IA, Alleva LM, Budd AC, and Cowden WB. 2008. Understanding the role of inflammatory cytokines in malaria and related diseases. Travel Med Infect Dis 6:67-81. 10.1016/j.tmaid.2007.07.002

Crompton PD, Moebius J, Portugal S, Waisberg M, Hart G, Garver LS, Miller LH, Barillas-Mury C, and Pierce SK. 2014. Malaria immunity in man and mosquito: insights into unsolved mysteries of a deadly infectious disease. Annu Rev Immunol 32:157-187. 10.1146/annurev-immunol-032713-120220

da Costa AG, Antonelli LR, Costa PA, Pimentel JP, Garcia NP, Tarrago AM, dos Santos Mdo P, Nogueira PA, Hekcmann MI, Sadahiro A, Teixeira-Carvalho A, Martins-Filho OA, and Malheiro A. 2014. The robust and modulated biomarker network elicited by the Plasmodium vivax infection is mainly mediated by the IL-6/IL-10 axis and is associated with the parasite load. J Immunol Res 2014:318250. 10.1155/2014/318250 
Deloron P, Roux Lombard P, Ringwald P, Wallon M, Niyongabo T, Aubry P, Dayer JM, and Peyron F. 1994. Plasma levels of TNF-alpha soluble receptors correlate with outcome in human falciparum malaria. Eur Cytokine Netw 5:331-336.

Doolan DL, Dobano C, and Baird JK. 2009. Acquired immunity to malaria. Clin Microbiol Rev 22:13-36, Table of Contents. 10.1128/CMR.00025-08

Espie E, Diene Sarr F, Diop F, Faye J, Richard V, Tall A, and Toure Balde A. 2015. Spatio-Temporal Variations in Malaria Incidence in Children Less than 10 Years Old, Health District of Sokone, Senegal, 20102013. PLoS One 10:e0137737. 10.1371/journal.pone.0137737

Farouk SE, Dolo A, Bereczky S, Kouriba B, Maiga B, Farnert A, Perlmann H, Hayano M, Montgomery SM, Doumbo OK, and Troye-Blomberg M. 2005. Different antibody- and cytokine-mediated responses to Plasmodium falciparum parasite in two sympatric ethnic tribes living in Mali. Microbes Infect 7:110-117. 10.1016/j.micinf.2004.09.012

Frosch AE, and John CC. 2012. Immunomodulation in Plasmodium falciparum malaria: experiments in nature and their conflicting implications for potential therapeutic agents. Expert Rev Anti Infect Ther 10:1343-1356. 10.1586/eri.12.118

Hu WC. 2013. Human immune responses to Plasmodium falciparum infection: molecular evidence for a suboptimal THalphabeta and TH17 bias over ideal and effective traditional TH1 immune response. Malar J 12:392. 10.1186/1475-2875-12-392

Jakobsen PH, Morris-Jones S, Theander TG, Hviid L, Hansen MB, Bendtzen K, Ridley RG, and Greenwood BM. 1994. Increased plasma levels of soluble IL-2R are associated with severe Plasmodium falciparum malaria. Clin Exp Immunol 96:98-103.

Kurtzhals JA, Adabayeri V, Goka BQ, Akanmori BD, Oliver-Commey JO, Nkrumah FK, Behr C, and Hviid L. 1998. Low plasma concentrations of interleukin 10 in severe malarial anaemia compared with cerebral and uncomplicated malaria. Lancet 351:1768-1772. 10.1016/S0140-6736(97)09439-7

Lyke KE, Burges R, Cissoko Y, Sangare L, Dao M, Diarra I, Kone A, Harley R, Plowe CV, Doumbo OK, and Sztein MB. 2004. Serum levels of the proinflammatory cytokines interleukin-1 beta (IL-1beta), IL6, IL-8, IL-10, tumor necrosis factor alpha, and IL-12(p70) in Malian children with severe Plasmodium falciparum malaria and matched uncomplicated malaria or healthy controls. Infect Immun 72:5630-5637. 10.1128/IAI.72.10.5630-5637.2004

MacMullin G, Mackenzie R, Lau R, Khang J, Zhang H, Rajwans N, Liles WC, and Pillai DR. 2012. Host immune response in returning travellers infected with malaria. Malar J 11:148. 10.1186/1475-2875-11148

Mahanta A, Kar SK, Kakati S, and Baruah S. 2015. Heightened inflammation in severe malaria is associated with decreased IL-10 expression levels and neutrophils. Innate Immun 21:546-552. $10.1177 / 1753425914561277$

Nussbaum JC, Van Dyken SJ, von Moltke J, Cheng LE, Mohapatra A, Molofsky AB, Thornton EE, Krummel MF, Chawla A, Liang HE, and Locksley RM. 2013. Type 2 innate lymphoid cells control eosinophil homeostasis. Nature 502:245-248. 10.1038/nature12526

Olliaro P. 2008. Editorial commentary: mortality associated with severe Plasmodium falciparum malaria increases with age. Clin Infect Dis 47:158-160. 10.1086/589288

Ong'echa JM, Davenport GC, Vulule JM, Hittner JB, and Perkins DJ. 2011. Identification of inflammatory biomarkers for pediatric malarial anemia severity using novel statistical methods. Infect Immun 79:4674-4680. 10.1128/IAI.05161-11

Perlmann H, Helmby H, Hagstedt M, Carlson J, Larsson PH, Troye-Blomberg M, and Perlmann P. 1994. IgE elevation and IgE anti-malarial antibodies in Plasmodium falciparum malaria: association of high IgE levels with cerebral malaria. Clin Exp Immunol 97:284-292. 
403

404

405

406

407

408

409

410

411

412

413

414

415

416

417

418

419

420

421

422

423

424

425

426

427

428

429

430

431

432

433

434

435

436

437

438

439

440

441

442

443

444

445

446

447

448

449

450

Perlmann P, Perlmann H, Flyg BW, Hagstedt M, Elghazali G, Worku S, Fernandez V, Rutta AS, and TroyeBlomberg M. 1997. Immunoglobulin E, a pathogenic factor in Plasmodium falciparum malaria. Infect Immun 65:116-121.

Peyron F, Burdin N, Ringwald P, Vuillez JP, Rousset F, and Banchereau J. 1994. High levels of circulating IL10 in human malaria. Clin Exp Immunol 95:300-303.

Pope SM, Brandt EB, Mishra A, Hogan SP, Zimmermann N, Matthaei KI, Foster PS, and Rothenberg ME. 2001. IL-13 induces eosinophil recruitment into the lung by an IL-5- and eotaxin-dependent mechanism. J Allergy Clin Immunol 108:594-601. 10.1067/mai.2001.118600

Porcherie A, Mathieu C, Peronet R, Schneider E, Claver J, Commere PH, Kiefer-Biasizzo H, Karasuyama H, Milon G, Dy M, Kinet JP, Louis J, Blank U, and Mecheri S. 2011. Critical role of the neutrophilassociated high-affinity receptor for IgE in the pathogenesis of experimental cerebral malaria. $J$ Exp Med 208:2225-2236. 10.1084/jem.20110845

Prakash D, Fesel C, Jain R, Cazenave PA, Mishra GC, and Pied S. 2006. Clusters of cytokines determine malaria severity in Plasmodium falciparum-infected patients from endemic areas of Central India. J Infect Dis 194:198-207. 10.1086/504720

Quelhas D, Puyol L, Quinto L, Nhampossa T, Serra-Casas E, Macete E, Aide P, Sanz S, Aponte JJ, Doolan DL, Alonso PL, Menendez C, and Dobano C. 2012. Intermittent preventive treatment with sulfadoxinepyrimethamine does not modify plasma cytokines and chemokines or intracellular cytokine responses to Plasmodium falciparum in Mozambican children. BMC Immunol 13:5. 10.1186/14712172-13-5

Queto T, Gaspar-Elsas MI, Masid-de-Brito D, Vasconcelos ZF, Ferraris FK, Penido C, Cunha FQ, Kanaoka Y, Lam BK, and Xavier-Elsas P. 2010. Cysteinyl-leukotriene type 1 receptors transduce a critical signal for the up-regulation of eosinophilopoiesis by interleukin-13 and eotaxin in murine bone marrow. J Leukoc Biol 87:885-893. 10.1189/jlb.1108709

Requena P, Campo JJ, Umbers AJ, Ome M, Wangnapi R, Barrios D, Robinson LJ, Samol P, Rosanas-Urgell A, Ubillos I, Mayor A, Lopez M, de Lazzari E, Arevalo-Herrera M, Fernandez-Becerra C, del Portillo H, Chitnis CE, Siba PM, Bardaji A, Mueller I, Rogerson S, Menendez C, and Dobano C. 2014. Pregnancy and malaria exposure are associated with changes in the B cell pool and in plasma eotaxin levels. J Immunol 193:2971-2983. 10.4049/jimmunol.1401037

Sarthou JL, Angel G, Aribot G, Rogier C, Dieye A, Toure Balde A, Diatta B, Seignot P, and Roussilhon C. 1997. Prognostic value of anti-Plasmodium falciparum-specific immunoglobulin G3, cytokines, and their soluble receptors in West African patients with severe malaria. Infect Immun 65:32713276.

Schofield L, and Grau GE. 2005. Immunological processes in malaria pathogenesis. Nat Rev Immunol 5:722-735. 10.1038/nri1686

Seka-Seka J, Brouh Y, Yapo-Crezoit AC, and Atseye NH. 2004. The role of serum immunoglobulin E in the pathogenesis of Plasmodium falciparum malaria in Ivorian children. Scand J Immunol 59:228-230.

Storm J, and Craig AG. 2014. Pathogenesis of cerebral malaria--inflammation and cytoadherence. Front Cell Infect Microbiol 4:100. 10.3389/fcimb.2014.00100

Thuma PE, van Dijk J, Bucala R, Debebe Z, Nekhai S, Kuddo T, Nouraie M, Weiss G, and Gordeuk VR. 2011. Distinct clinical and immunologic profiles in severe malarial anemia and cerebral malaria in Zambia. J Infect Dis 203:211-219. 10.1093/infdis/jiq041

Torrentino-Madamet M, Fall B, Benoit N, Camara C, Amalvict R, Fall M, Dionne P, Ba Fall K, Nakoulima A, Diatta B, Dieme Y, Menard D, Wade B, and Pradines B. 2014. Limited polymorphisms in $\mathrm{k} 13$ gene in Plasmodium falciparum isolates from Dakar, Senegal in 2012-2013. Malar J 13:472. 10.1186/1475-2875-13-472

Trape JF, Tall A, Sokhna C, Ly AB, Diagne N, Ndiath O, Mazenot C, Richard V, Badiane A, Dieye-Ba F, Faye J, Ndiaye G, Diene Sarr F, Roucher C, Bouganali C, Bassene H, Toure-Balde A, Roussilhon C, Perraut 
451

452

453

454

455

456

457

458

459

460

461

462

463

464

465

466
R, Spiegel A, Sarthou JL, da Silva LP, Mercereau-Puijalon O, Druilhe P, and Rogier C. 2014. The rise and fall of malaria in a West African rural community, Dielmo, Senegal, from 1990 to 2012: a 22 year longitudinal study. Lancet Infect Dis 14:476-488. 10.1016/S1473-3099(14)70712-1

Walther $\mathrm{M}$, Tongren JE, Andrews L, Korbel D, King E, Fletcher $\mathrm{H}$, Andersen RF, Bejon $\mathrm{P}$, Thompson $\mathrm{F}$, Dunachie SJ, Edele F, de Souza JB, Sinden RE, Gilbert SC, Riley EM, and Hill AV. 2005. Upregulation of TGF-beta, FOXP3, and CD4+CD25+ regulatory T cells correlates with more rapid parasite growth in human malaria infection. Immunity 23:287-296. 10.1016/j.immuni.2005.08.006

Wang TN, Chiang W, Tseng HI, Chu YT, Chen WY, Shih NH, and Ko YC. 2007. The polymorphisms of Eotaxin 1 and CCR3 genes influence on serum IgE, Eotaxin levels and mild asthmatic children in Taiwan. Allergy 62:1125-1130. 10.1111/j.1398-9995.2007.01485.x

White NJ, Pukrittayakamee S, Hien TT, Faiz MA, Mokuolu OA, and Dondorp AM. 2014. Malaria. Lancet 383:723-735. 10.1016/S0140-6736(13)60024-0

WHO. 2000. Severe falciparum malaria. Trans R Soc Trop Med Hyg 94 (Suppl1):1-90.

WHO. 2015. World Malaria Report 2015. Geneva, Switzerland: World Health Organization. 


\section{Table 1 (on next page)}

Demographic, clinical, and disease outcome data. 


\begin{tabular}{|c|c|c|c|c|c|}
\hline & & CT & NCM & CM & Total \\
\hline \multirow{2}{*}{ Gender } & Male & 11 & 11 & 22 & 44 \\
\hline & Female & 7 & 6 & 5 & 17 \\
\hline \multirow{2}{*}{ Age } & Range & $23-57$ & $5-74$ & $15-80$ & $5-80$ \\
\hline & Median & 28.5 & 18 & 26 & 26 \\
\hline \multirow{2}{*}{ HB } & Normal & 18 & 17 & 11 & 46 \\
\hline & Low & 0 & 0 & 16 & 16 \\
\hline \multirow{2}{*}{ Parasitemia } & Median & 0 & 520 & 1452 & $1022^{a}$ \\
\hline & IQR & 0 & 2552 & 9981 & $7654^{a}$ \\
\hline \multirow{2}{*}{ Outcome } & Survived & 18 & 17 & 18 & 53 \\
\hline & Deceased & 0 & 0 & 9 & 9 \\
\hline \multirow{6}{*}{$\begin{array}{l}\text { Organ } \\
\text { Failure }\end{array}$} & Neurological & 0 & 0 & 27 & 27 \\
\hline & Respiratory & 0 & 0 & 6 & 6 \\
\hline & Kidney & 0 & 0 & 13 & 13 \\
\hline & Liver & 0 & 0 & 8 & 8 \\
\hline & Hematologic & 0 & 0 & 7 & 7 \\
\hline & Hemodynamic & 0 & 0 & 2 & 2 \\
\hline
\end{tabular}

$\mathrm{CT}$, control individuals; NCM, non-cerebral malaria patients; CM, cerebral malaria patients; HB, hemoglobin (Low, < 100 g/L; Normal, > 100 g/L); IQR, Inter Quartile Range. ${ }^{a}$, value for NCM $+\mathrm{CM}$. 


\section{Table 2 (on next page)}

Biomarkers significantly differing between non-cerebral and cerebral malaria patients after multiple testing adjustment. -> 


\begin{tabular}{cccc}
\hline Biomarkers & MW P values & BH critical values & Significance \\
\hline TNFa $^{\text {a }}$ & 0.049 & 0.017 & No \\
IL-15 $^{\mathrm{a}}$ & 0.042 & 0.013 & No \\
MCP-1 $^{\mathrm{a}}$ & 0.013 & 0.010 & No \\
IL-6 $^{\mathrm{a}}$ & 0.008 & 0.008 & Yes \\
IL-1RA $_{\text {IL-5 }}^{\mathrm{b}}$ & 0.007 & 0.006 & Yes \\
IL-8 $^{\mathrm{a}}$ & 0.002 & 0.004 & Yes \\
\hline
\end{tabular}

MW, Mann-Whitney U comparison; BH, Benjamini-Hochberg correction; a, increased in $\mathrm{CM}$; ${ }^{\mathrm{b}}$, decreased in $\mathrm{CM}$. 


\section{Table 3 (on next page)}

Biomarkers significantly increased in deceased of cerebral malaria compared to survivors. agment4s 2 o 3 


\begin{tabular}{cccc}
\hline Biomarkers & MW P values & BH critical values & Significance \\
\hline IL-6 & 0.027 & 0.015 & No \\
IL-8 & 0.017 & 0.010 & No \\
Eotaxin & 0.007 & 0.008 & Yes \\
TNF $\alpha$ & 0.003 & 0.006 & Yes \\
IL-15 & 0.002 & 0.004 & Yes \\
MCP-1 & 0.001 & 0.002 & Yes \\
\hline
\end{tabular}

MW, Mann-Whitney U comparison; BH, Benjamini-Hochberg correction.

1 


\section{Table 4(on next page)}

Biomarkers correlated with kidney failure in cerebral malaria patients. 


\begin{tabular}{|c|c|c|c|c|c|}
\hline & $\rho$ ( $p$ value $)$ & & $\rho$ ( $p$ value $)$ & & $\rho(p$ value $)$ \\
\hline Eotaxin & $0.514(0.006)$ & IL-10 & $0.457(0.017)$ & IL-1 $\alpha$ & $0.593(0.001)$ \\
\hline G-CSF & $0.500(0.008)$ & IL-12p70 & $0.445(0.020)$ & IP-10 & $0.533(0.004)$ \\
\hline GM-CSF & $0.714(<0.001)$ & IL-15 & $0.621(0.001)$ & MCP-1 & $0.581(0.002)$ \\
\hline IFN $\alpha 2$ & $0.525(0.005)$ & IL-17A & $0.401(0.038)$ & TNF $\alpha$ & $0.542(0.003)$ \\
\hline IFN $\gamma$ & $0.529(0.005)$ & IL-1RA & $0.390(0.044)$ & & \\
\hline
\end{tabular}

$\rho$, Spearman's Rho coefficient.

1 


\section{Table 5 (on next page)}

Variation of biomarker levels between admission and release from hospital in cerebral malaria patients. 


\begin{tabular}{cccc}
\hline & $\begin{array}{c}\text { Admission (pg/ml) } \\
\text { MN } \pm \text { SD / MD }\end{array}$ & $\begin{array}{c}\text { Release (pg/ml) } \\
\text { MN } \pm \text { SD / MD }\end{array}$ & P value \\
\hline G-CSF & $651 \pm 1869 / 163$ & $116 \pm 111 / 99$ & 0.007 \\
IL-10 & $1848 \pm 1749 / 464$ & $99 \pm 165 / 40$ & 0.002 \\
TNF $\alpha$ & $90 \pm 101 / 60$ & $24 \pm 14 / 20$ & 0.002 \\
IL-1 $\alpha$ & $121 \pm 165 / 76$ & $57 \pm 80 / 28$ & 0.013 \\
IL-8 & $213 \pm 417 / 66$ & $58 \pm 152 / 14$ & 0.001 \\
IP-10 & $5668 \pm 6297 / 2496$ & $1757 \pm 2191 / 892$ & 0.002 \\
MCP-1 & $1303 \pm 1754 / 508$ & $409 \pm 678 / 242$ & 0.005 \\
\hline
\end{tabular}

Admission, biomarker levels at the time of hospital admission of CM patients; Release, biomarker levels at the time of release of $\mathrm{CM}$ patients from hospital; $P$ value, two-tailed 1 $p$ value of a Wilcoxon Rank test; MN, mean; SD, standard deviation; MD, median. 


\section{1}

Serum levels of immune mediators during malaria. Root s2? g 3

The levels of 29 biomarkers were measured in control subjects (CT) and in non-cerebral (NCM) and cerebral (CM) malaria patients. The median value of each cytokine in the global population (CT + NCM + CM) was used as a cut-off value to determine the percentage of "high" (above median) biomarker producer individuals in each group. The ascendant biomarker profile of the $\mathrm{CT}(\mathrm{A})$ was determined and the resulting curve used as a reference to visualize the difference in the proportion of high biomarker producers with the NCM (B) and $\mathrm{CM}(\mathrm{C})$ groups. Hatched bars represent biomarkers for which there is a significant difference in Mann-Whitney $\mathrm{U}$ pairwise comparison with the CT reference group after Benjamini-Hochberg multiple test adjustment. 

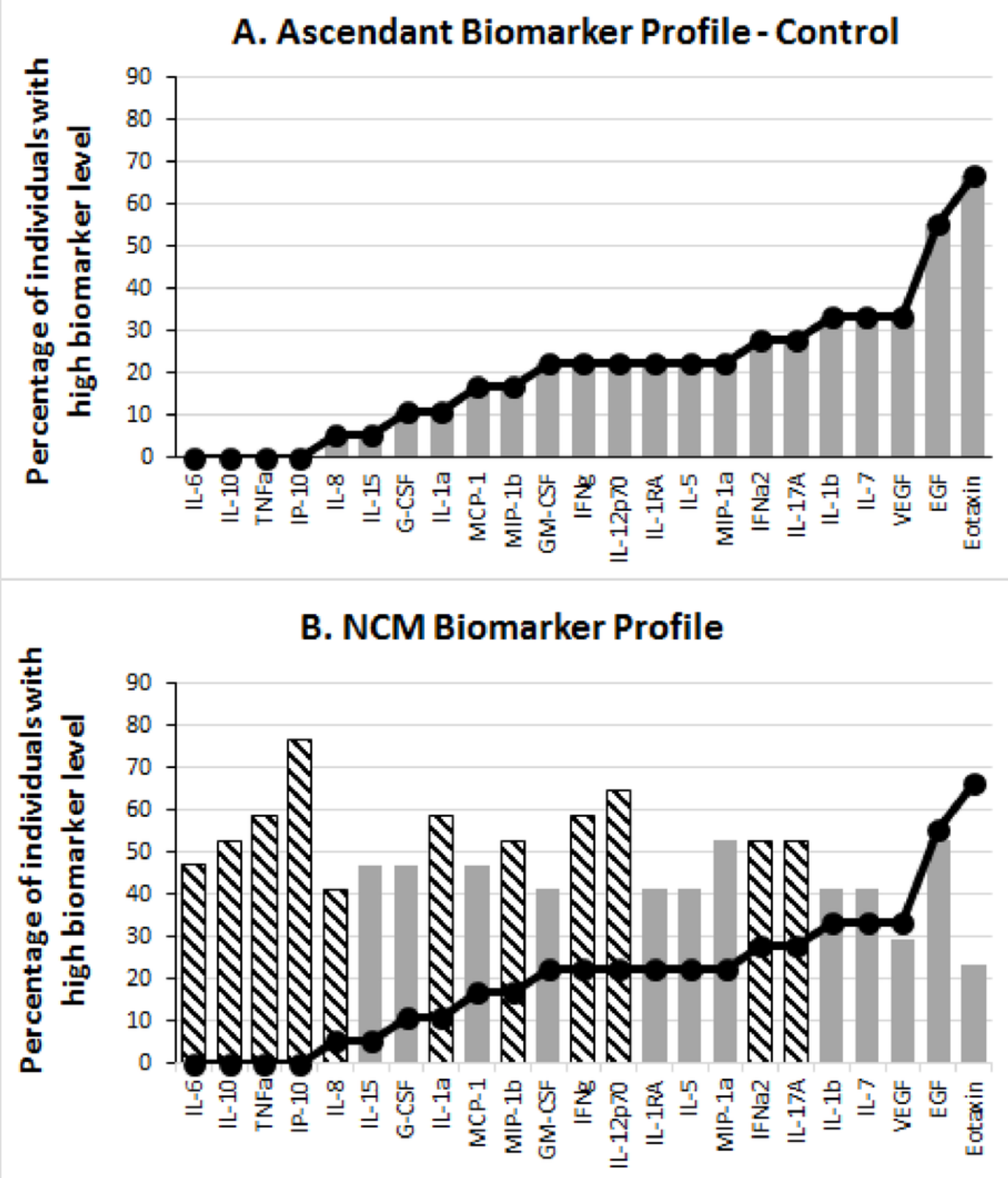

\section{CM Biomarker Profile}

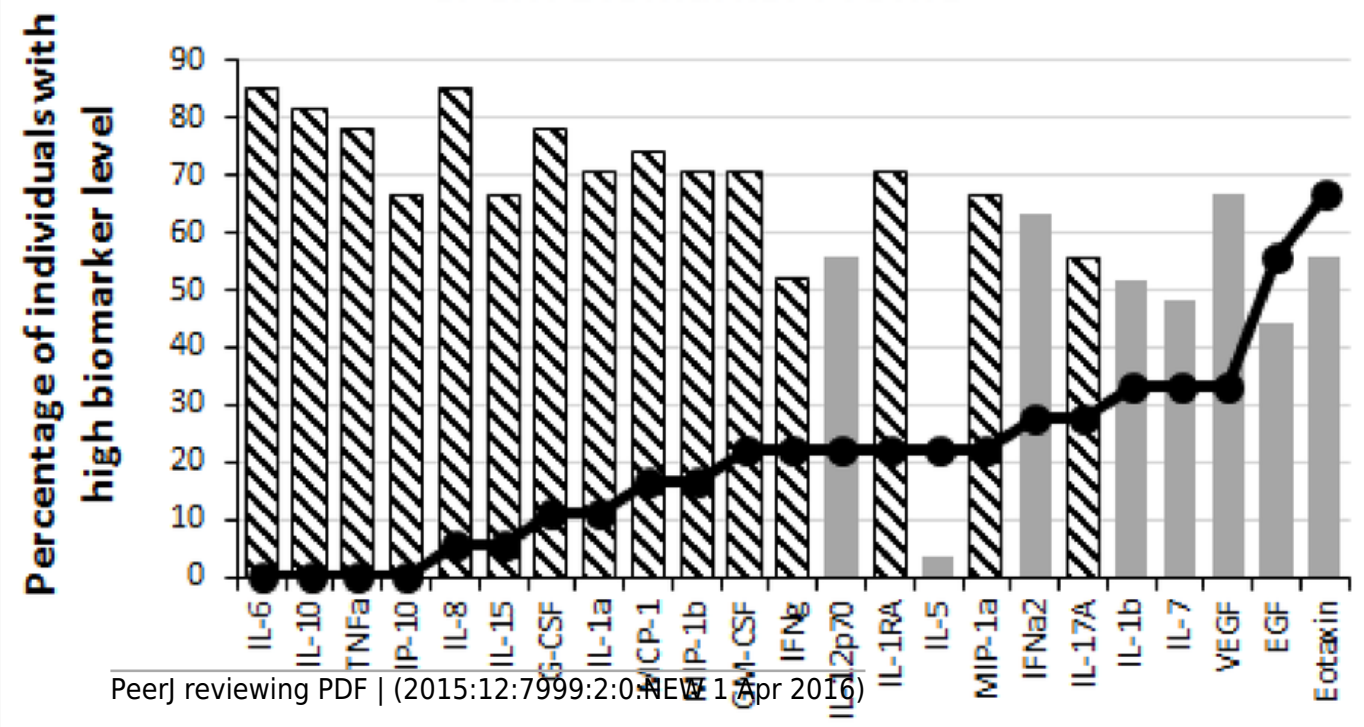




\section{Figure 2 (on next page)}

Serum biomarker levels in control individuals and in non-cerebral and cerebral malaria patients. io:s2? W॰

Biomarkers that significantly differed across the three groups in Kruskal-Wallis test after Benjamini-Hochberg adjustment are shown. Box plots represent medians with $25^{\text {th }}$ and $75^{\text {th }}$ percentiles, bars $10^{\text {th }}$ and $90^{\text {th }}$ percentiles, and dots outliers for biomarker concentrations. $P$, $\mathrm{p}[i]$ values in Kruskal-Wallis tests. C, critical values in Benjamini-Hochberg correction. io:s2? W 

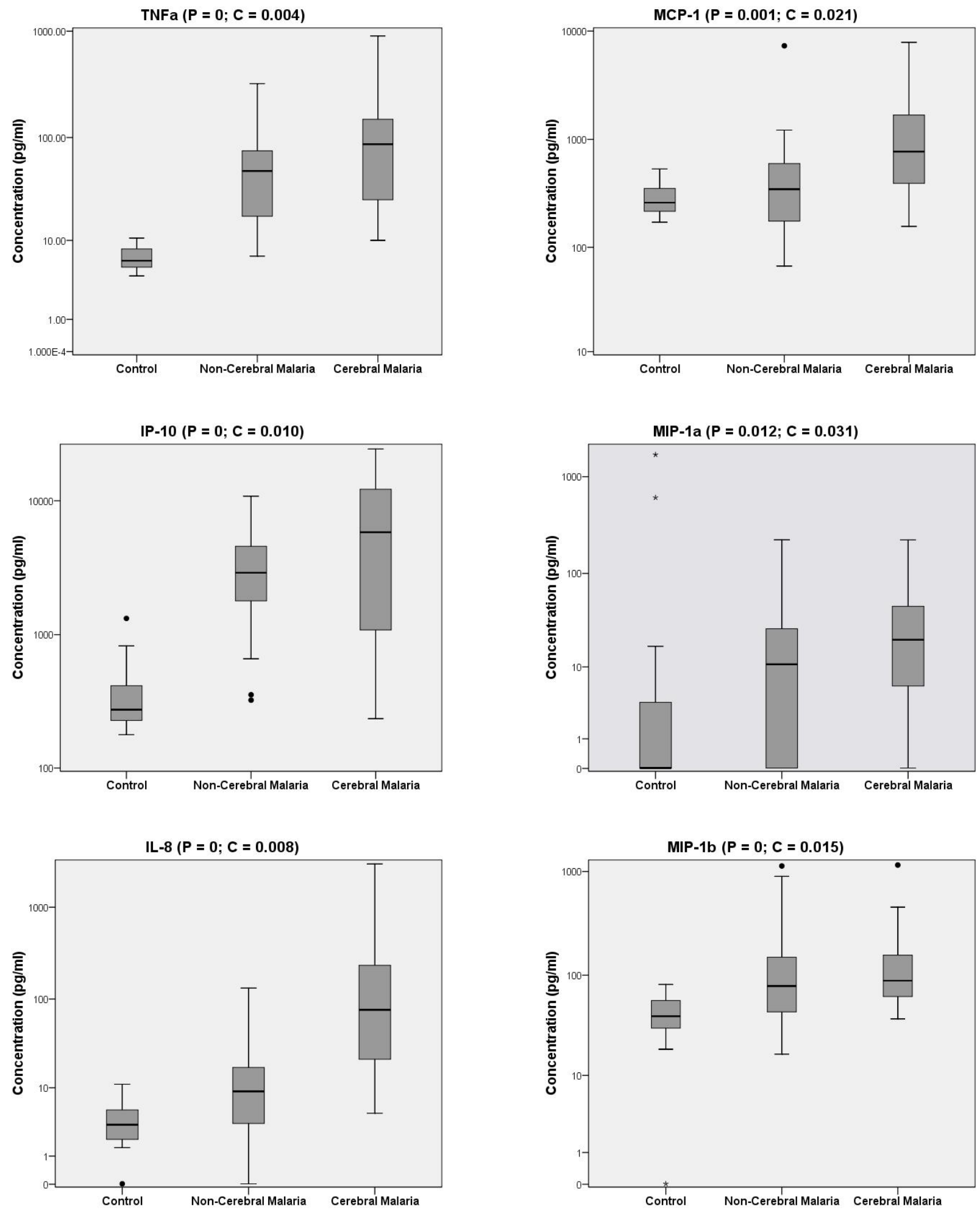

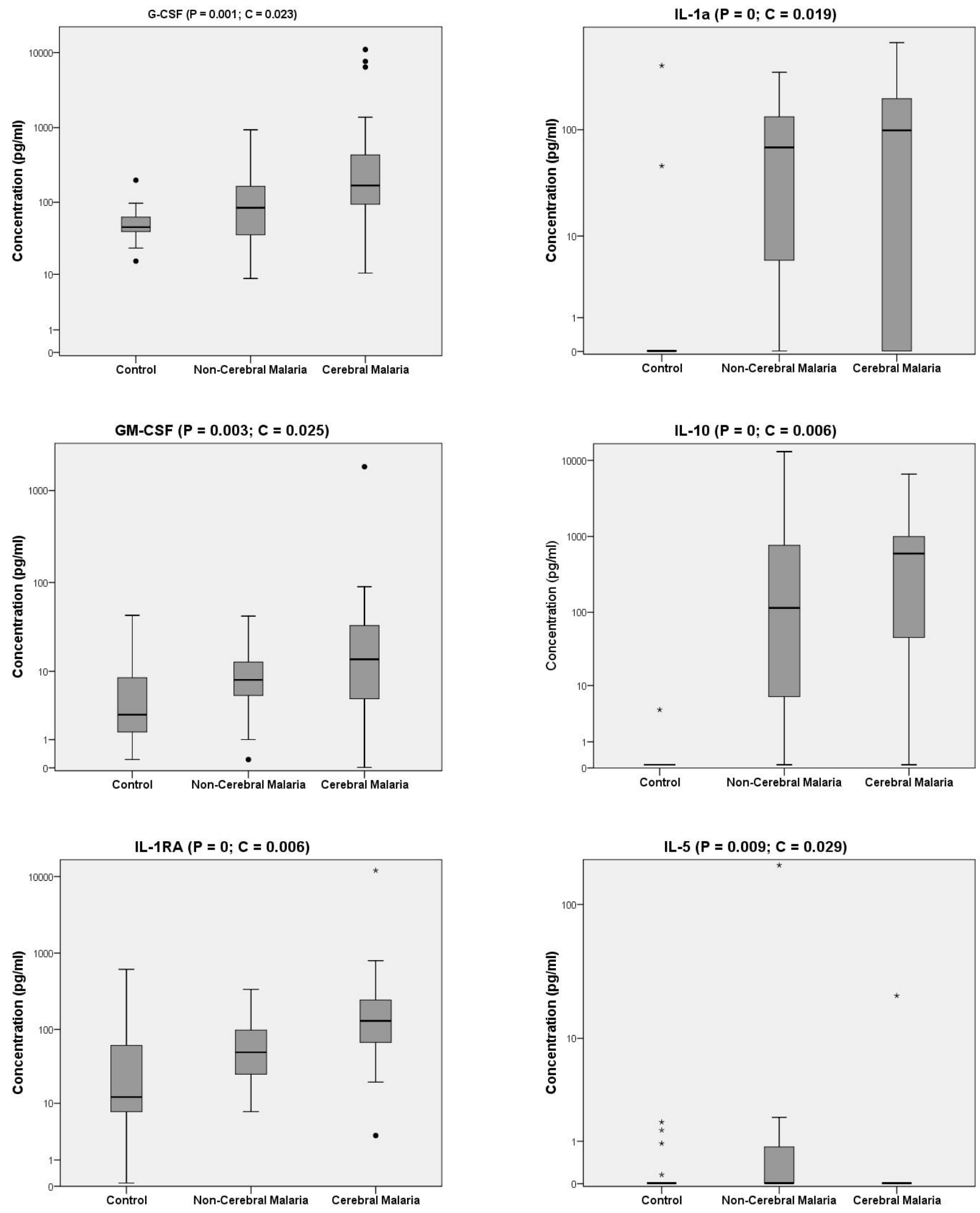

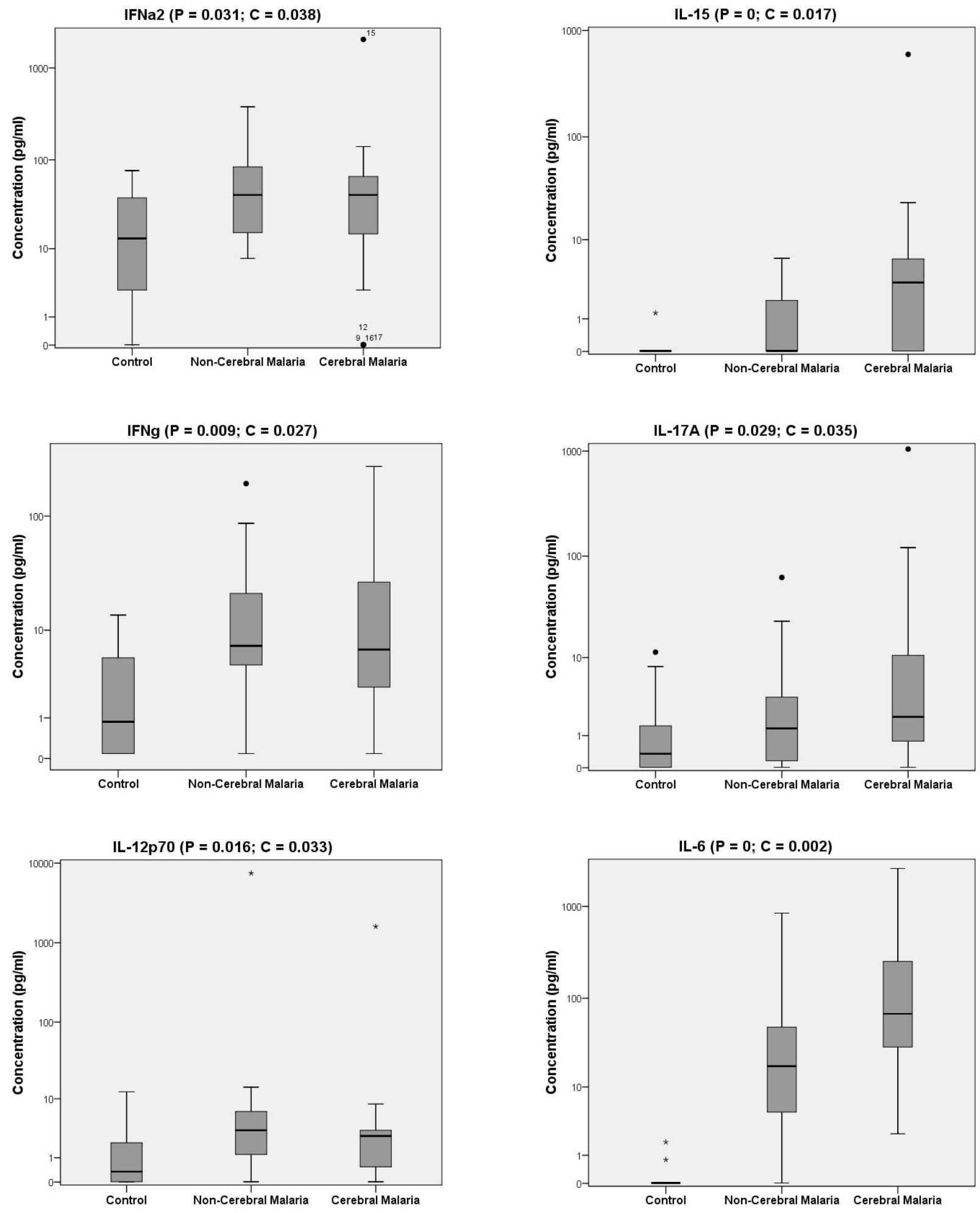


\section{3}

Levels of inflammatory immune mediators are higher in cerebral than in non-cerebral malaria patients.

The ascendant biomarker profile curve of the NCM (line) was plotted on the CM graph (bars) to visualize the difference in the proportion of high biomarker producers. Hatched bars represent biomarkers for which there is a significant difference in Mann-Whitney $U$ pairwise comparison between the two groups after Benjamini-Hochberg multiple test adjustment.

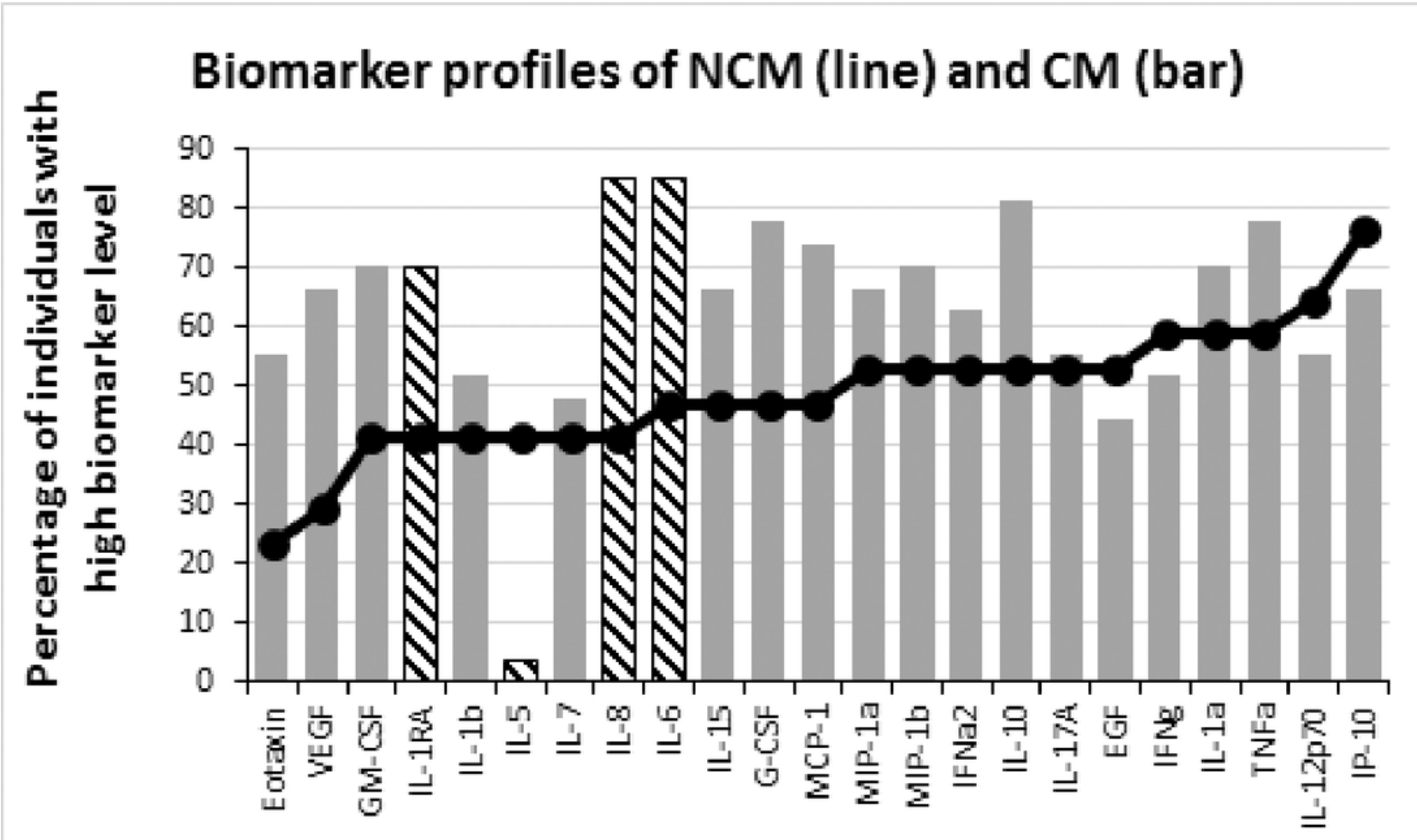


4

Levels of inflammatory immune mediators are higher in deceased than in survivors of cerebral malaria.

The ascendant biomarker profile curve of the survivors (line) was plotted on the deceased graph (bars) to visualize the difference in the proportion of high biomarker producers. Hatched bars represent biomarkers for which there is a significant difference in MannWhitney $U$ pairwise comparison between the two groups after Benjamini-Hochberg multiple test adjustment.

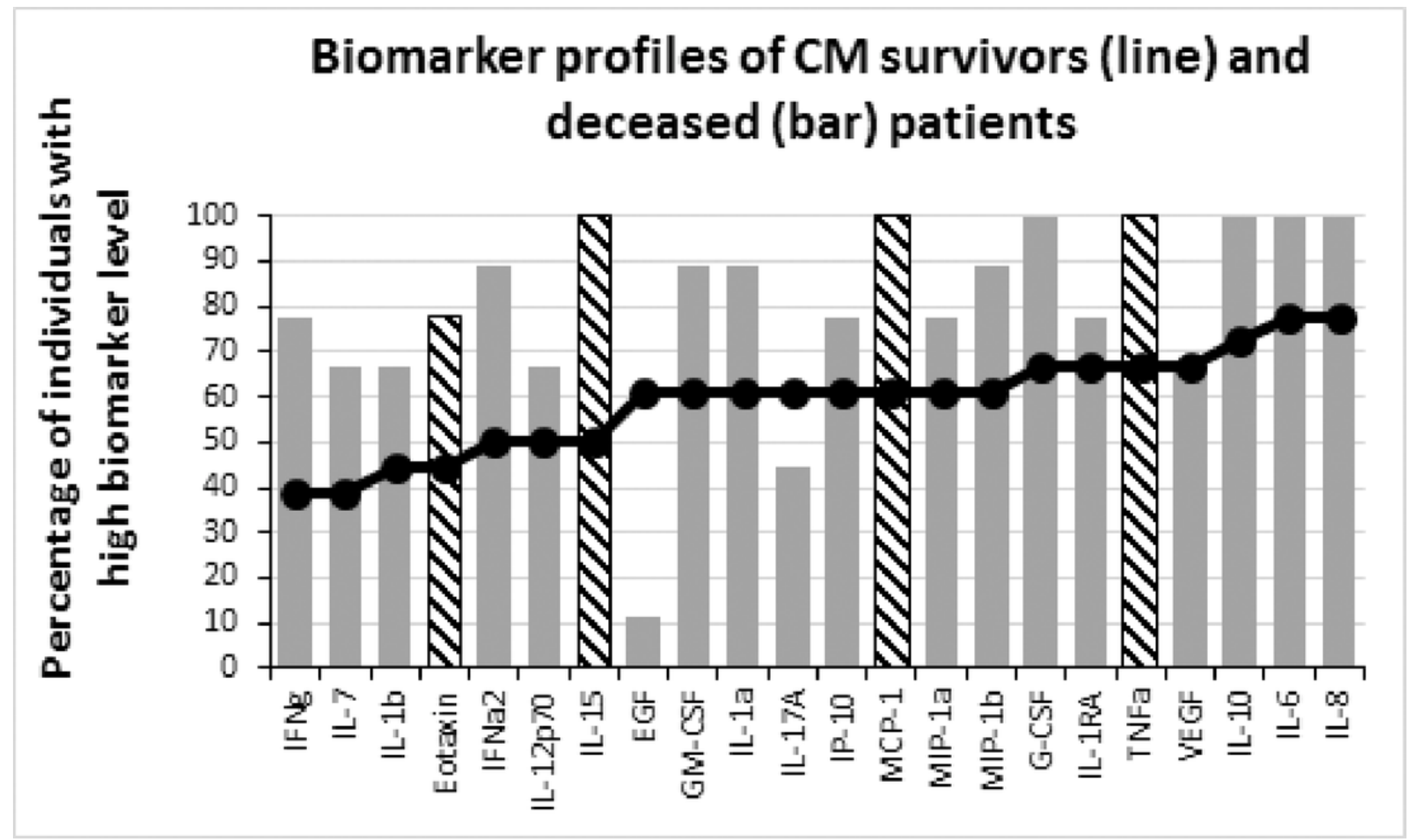

